\title{
Design Tactile Interfaces with Enhanced Depth Images With Patterns and Textures for Visually Impaired People
}

\author{
George Kokkonis ${ }^{1}$, Kostas Psannis ${ }^{1}$, Christodoulos Asiminidis ${ }^{2}$, Sotirios Kontogiannis ${ }^{2}$ \\ ${ }^{1}$ Department of Applied Informatics, University of Macedonia, Thessaloniki, Greece \\ ${ }^{2}$ Laboratory Team of Distributed Microcomputer Systems, Department of Mathematics, \\ ${ }^{2}$ University of Ioannina, Ioannina, Greece
}

\begin{abstract}
This paper presents the design and development of Tactile Interfaces with Enhanced Images with Patterns and Textures for Visually Impaired People. In particular, haptic interfaces uses a number of predefine tactile texture and patterns to enhance the experience of the tactile feeling of images for visually impaired people. The open source OpenCv application is used to automatically change group of colours from depth images to tactile texture and patterns. The depth images are captured with the he $\lambda \mathrm{p}$ of RGB-Depth cameras. The tactile images are then explored by visually impaired people with the use of haptic interfaces. The predefined textures and patterns are more easily distinguished than uniformly colors in depth images.
\end{abstract}

Key Words: Haptics, tactile interfaces, 3D models, depth images, RGB-Depth cameras, haptic geometric patterns

\section{INTRODUCTION}

Touch is a basic sensation for the understanding of our surrounding environment. The sensation of touch involves the skin's ability to separate the surface properties of an object, and the kinaesthetic ability of the muscles to determine the body's position.

The reaction that we acquire whenever we explore the shape, the texture, the geometry, the mass, the elasticity, and the dimensions of objects with our hands is called tactile feedback. There is also the kinaesthetic feedback which is the stimuli that is produced in muscles and tendons from power-force translation, limb motion control, and body position.

Visually impaired people are depending in the tactile and kinaesthetic feedback to explore their surroundings. RGB-Depth cameras can record the $3 \mathrm{~d}$ model of the environment and replicate it to a $3 \mathrm{~d}$ virtual reality. Haptic interfaces can be used by visually impaired people to explore and react with this augmented virtuality. Geometric patterns can be used to increase the experience of the haptic interface user and enhance the haptic feeling of the $3 \mathrm{~d}$ objects.

The rest of the paper is organized as follows: Section 2 presents a related work on tactile interfaces. Section 3 presents the $3 \mathrm{D}$ depth monitoring technology that is used to capture and reproduce the $3 \mathrm{D}$ virtual model of the surroundings. Section 4 proposes the geometric patterns that could be used for the tactile enhancement of the 3D models. Section 5 describes the open OpenCV algorithm that manipulates the $3 \mathrm{~d}$ models to reproduce the enhanced tactile $3 d$ objects. Section 6 describes the H3D haptic program that transforms the patterned image to haptic objects. Section 7 , finally concludes the paper.

\section{HAPTIC DEVICES}

Virtual objects acquire realistic properties such as mass, hardness, texture and elasticity. The tactile interface is an interactive actuator that interacts with the $3 \mathrm{D}$ object and the user in a virtual environment. It receives commands from the user and manipulates the $3 \mathrm{D}$ virtual objects. It consequently receives feedback from the virtual environment and creates stimuli on the user's skin and muscles.

The tactile interfaces are split into two categories according to the stimuli that they produce to the users. They are divided into tactile interfaces when the stimuli that they produce refers to the skin of the user and to kinesthetic haptic interface when the stimuli refers to muscle of the user and his/her body position. 
Tactile Interface are more common and they support skin feedback and produce vibration, pressure and heat to the users fingertips. They help the user to explore elasticity, the texture and the dimensions of virtual 3D objects.

Kinesthetic haptic devices provide feedback to user according to his movements and his body position. They help the user to explore the mass, the friction and the hardness of the virtual 3D objects.

One commonly used haptic devices, is the "3D Systems Touch Haptic Device" (1), figure 1. It offers both tactile and kinesthetic feedback to the user. It is a haptic device that user a ring or a stylus to interact with the user. It measures the position of the user's fingertip. The user can feel the sense of touch, movement constraints, friction and surface texture, and other features of virtual objects.

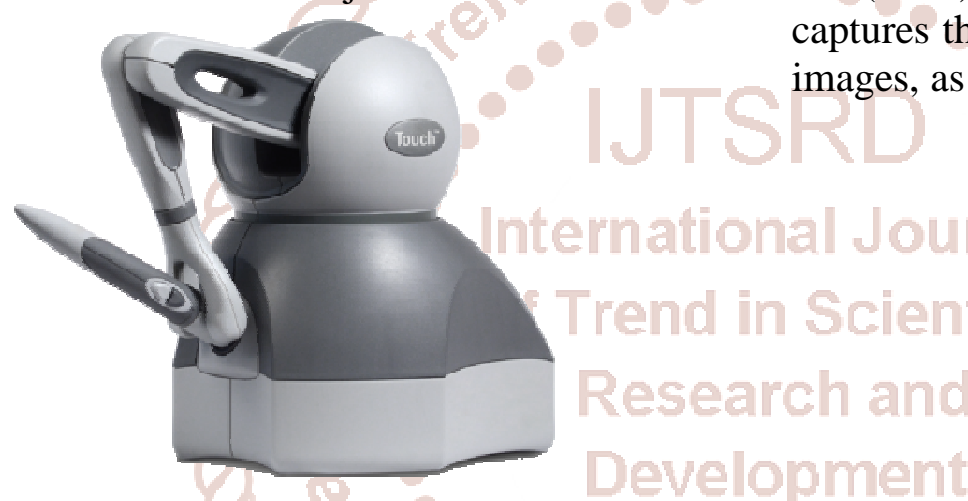

Figure1. 3D Systems Touch Haptic Device

Another common haptic device is the "Novint Falcon" (2). It was released in 2007 and it was the first haptic device that offered high resolution 3D feedback to the user, Figure 2.

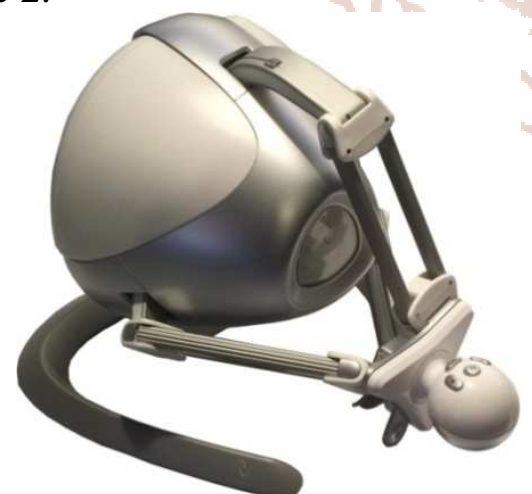

Figure2. Novint Falcon Haptic Device

The 3D Systems Touch Haptic Device offers six Degrees Of Freedom (DOF) to the user while the Novint Falcon offers 3 DOF. Both devices uses $1 \mathrm{kHz}$ servo rate, which means that they can produce 1000 update packets per second.

\section{DEPTH CAMERAS FOR 3D MODELLING}

In order to replicate real world objects to 3D virtual objects, RGB-depth cameras are used. One low cost RGB-depth camera that is commonly used is the Microsoft Kinect Depth sensor (3). It is composed by a VGA video camera, a CMOS depth sensor and an infrared projector. The projector emits infrared light to the physical object. The physical object reflects the infrared light back to the CMOS depth sensor and the depth sensor captures the reflected infrared light. The time passed from the moment the projector emits the light until the light reaches the depth sensor defines the distance between the sensor and the physical object. This time is called "time of flight".

In order to connect the kinect sensor to the $\mathrm{pc}$ and produce custom software for 3D-Depth mapping, Microsoft provides the Kinect Software Development Kit (SDK) and the Kinect Studio. The kinect studio captures the 3D image, the VGA image and the depth images, as shown in Figure 3.

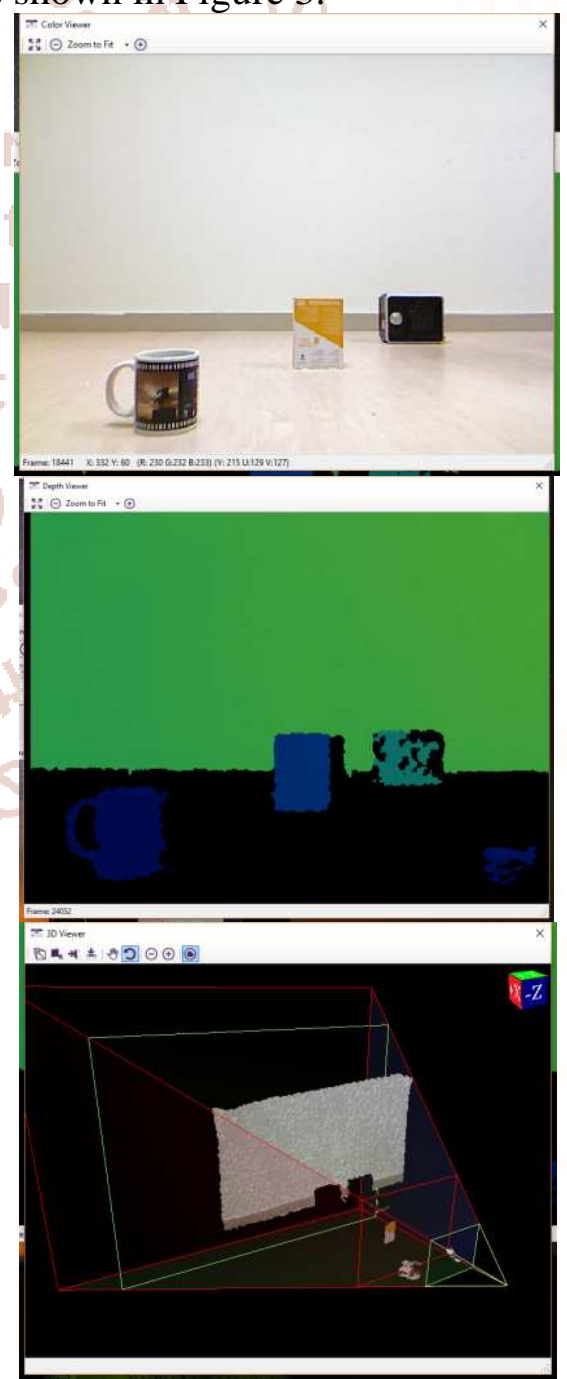

Figure3. Images captured from the Kinect sensor. The VGA image at the left side, the Depth image at the middle and the 3D image at the right 


\section{MAPPING DEPTH IMAGES TO ENHANCED PATTERNED IMAGES.}

In order these images to be more easily haptically explored the depth images of figure 3 should be transformed to a grey scale image as shown in Figure4.

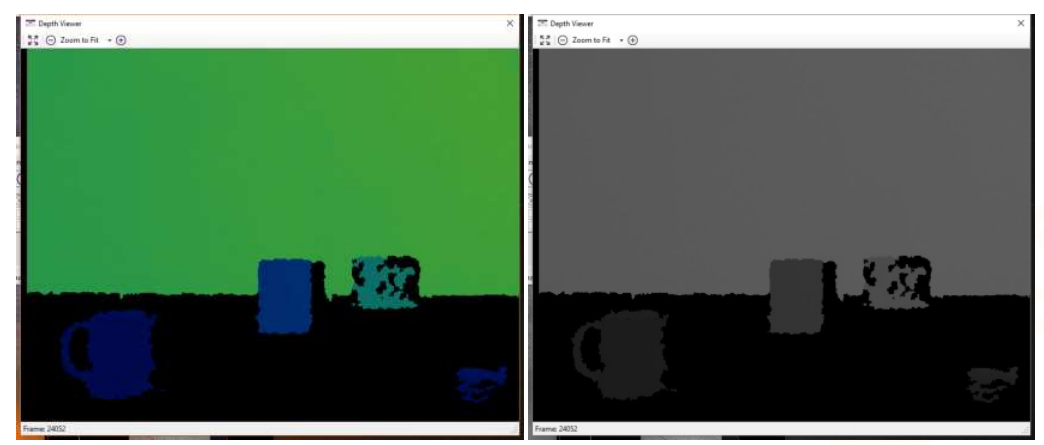

Figure4.Depth images transformed to gray scale images

Let us assume that we have an 11 RGB Grey scale. as depicted in figure 5.

\begin{tabular}{|c|c|c|c|c|c|}
\hline No. & Red & Green & Blue & RGB \% & Hex Color Code \\
\hline 1 & 255 & 255 & 255 & $(100,100,100)$ & FFFFFF \\
\hline 2 & 229 & 229 & 229 & $(90,90,90)$ & E5E5E5 \\
\hline 3 & 204 & 204 & 204 & $(80,80,80)$ & CCCCCC \\
\hline 4 & 178 & 178 & 178 & $(70,70,70)$ & B2B2B2 \\
\hline 5 & 153 & 153 & 153 & $(60,60,60)$ & 999999 \\
\hline 6 & 127 & 127 & 127 & $(50,50,50)$ & 7F7F7F \\
\hline 7 & 102 & 102 & 102 & $(40,40,40)$ & 666666 \\
\hline 8 & 76 & 76 & 76 & $(30,30,30)$ & 4 C4C4C \\
\hline 9 & 51 & 51 & 51 & $(20,20,20)$ & 333333 \\
\hline 10 & 25 & 25 & 25 & $(10,10,10)$ & 191919 \\
\hline 11 & 0 & 0 & 0 & $(0,0,0)$ & 000000 \\
\hline
\end{tabular}

Figure5. Gray scale in the RGB model

These 11 Grey levels should be corresponded to the simple geometrical patterns, such as those of Figure 6 .
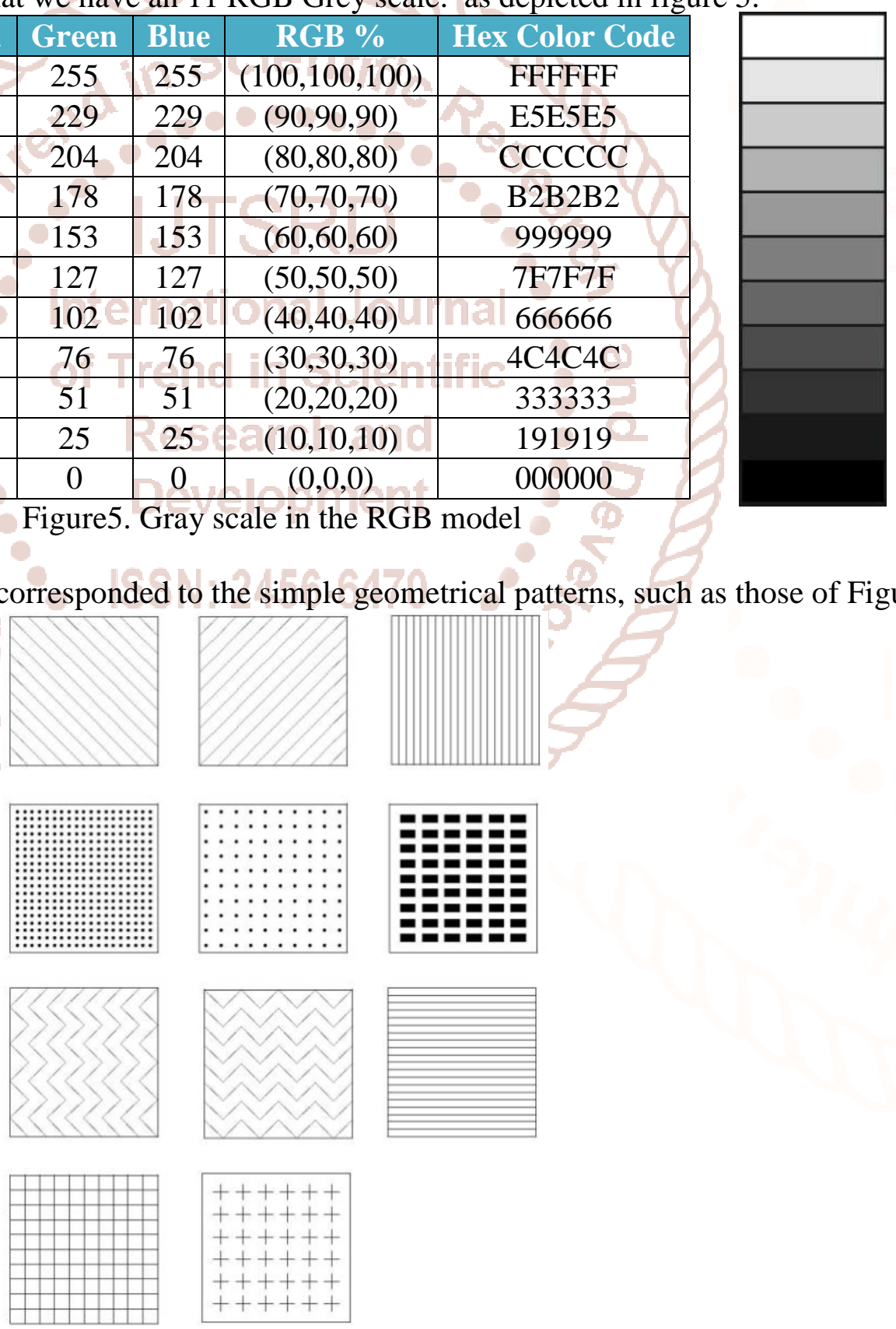
The image of Figure 4 is then transformed in Figure 7.

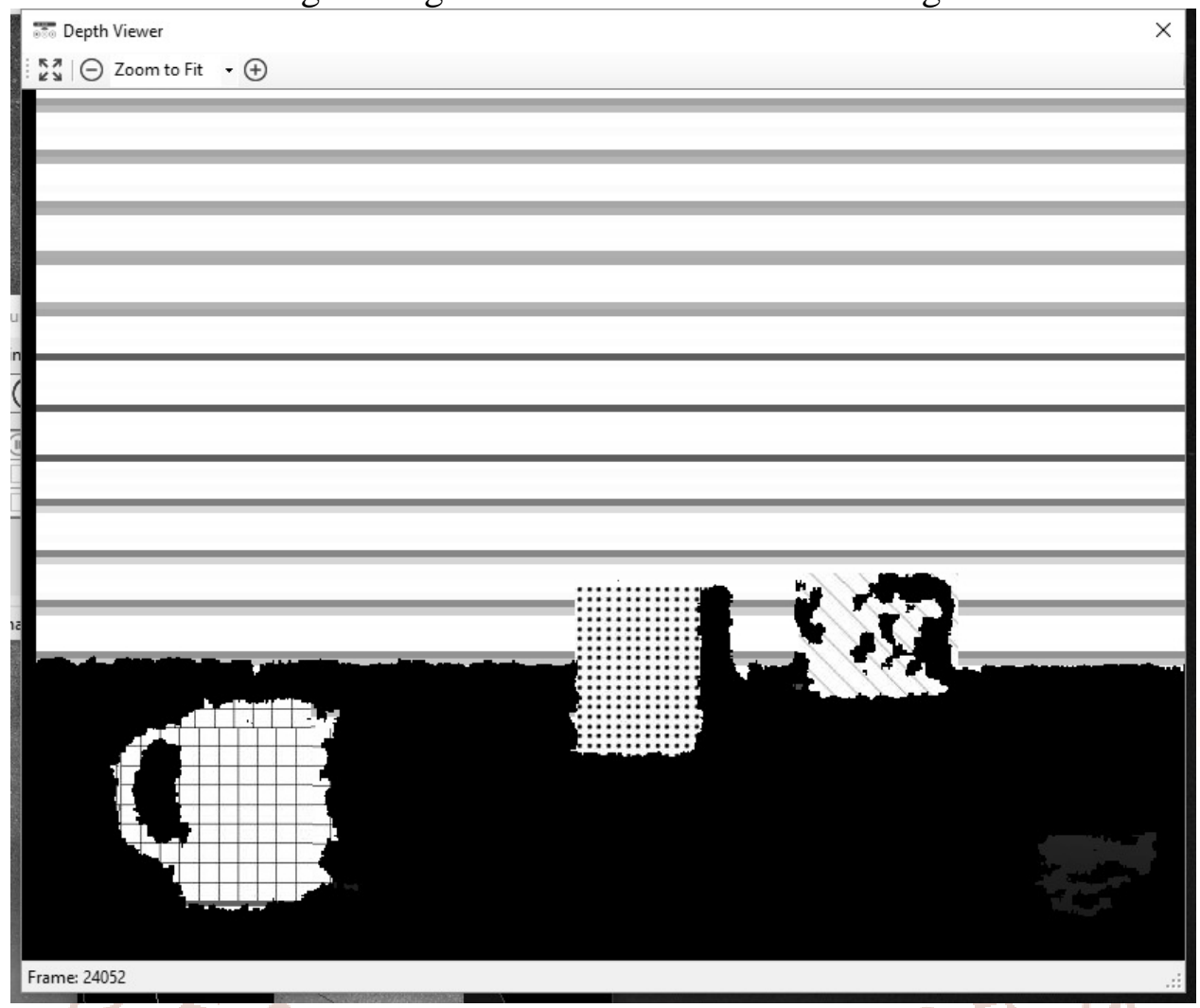

Figure7. Haptically enhanced depth image

5. THE USE OF OPENCV FOR AUTOMATIC TRANSFORMATION OF RGB IMAGES TO HAPTIC IMAGES

This transformation can automatically be done with the help of the OpenCV (4) image processing library and python.

The imread function will load the colored RGB image to OpenCV. Next, we convert the color image to grayscale with the help of the cvtColor function.

In order to convert the original image from the RGB color space to gray, the code COLOR_BGR2GRAY is used.

$$
\text { Gray }=\text { cv2.cvtColor }
$$

(Image, cv2.COLOR_BGR2GRAY)

Now we have to correspond the levels of the gray scale of Figure 5 to the geometrical patterns of Figure 6. This is done with the help of the library colorspace.

\section{TRANSFORM GRAYSCALE IMAGES TO HAPTIC OBJECTS}

In order to haptically explore the patterned images produced in the previous section the H3D haptic application is used (5). The H3D uses XML files to pass haptic properties to the objects illustrated in the pictures. The static friction, the dynamic friction, the stiffness and the maximum height of the objects can be given to objects with the following XmL code.

$<$ DepthMapSurface stiffness="0.6" maxDepth="0.008"

staticFriction $=" 0.9 "$

dynamicFriction $=" 0.9$ "

whiteIsOut $="$ False" >

<ImageTexture containerField="depthMap" url="patterned_image.png" repeatS="false"

repeat $T=$ "false"/>

</DepthMapSurface>

\section{CONCLUSION}

The system proposed gives the ability to visually impaired people to haptically explore images and the surrounding environment with the help of tactile interfaces. Predefined patterns and textures are used to enhance the experience of the user. Predefined textures and patterns help visually impaired people to distinguish more easily the objects that are included in a picture. The color of the object is changed automatically with the help of the OpenCv application to the predefined patterns. These patterns are more easily distinguished by haptic interfaces than uniformly colors. 
International Journal of Trend in Scientific Research and Development (IJTSRD) ISSN: 2456-6470

REFERENCES

1. Touch.,

3D

Systems. https://www.3dsystems.com/haptics-devices/touch. [Online] Dec 2018.

2. N. Karbasizadeh, A. Aflakiyan, M. Zarei, M. T. Masouleh and A. Kalhor. Dynamic identification of the Novint Falcon Haptic device. . 4th International Conference on Robotics and Mechatronics (ICROM), Tehran. 2016, pp. 518523.

3. Zhang, Z. Microsoft kinect sensor and its effect. IEEE multimedia, . Vol. 19(2), 4-10.
4. Jeeva, B., Sanjay, V., Purohit, V., Tauro, D. O., \& Vinay, J. Design and Development of Automated Intelligent Robot Using OpenCV. In 2018 International Conference on Design Innovations for 3Cs Compute Communicate Control (ICDI3C) . pp. 92-96.

5. Aondoakaa, A. S., Swash, R. M., \& Sadka, A. 3D depth estimation from a holoscopic 3D image. In 3D Image Acquisition and Display: Technology, Perception and Applications. 2017, DW1F-5.

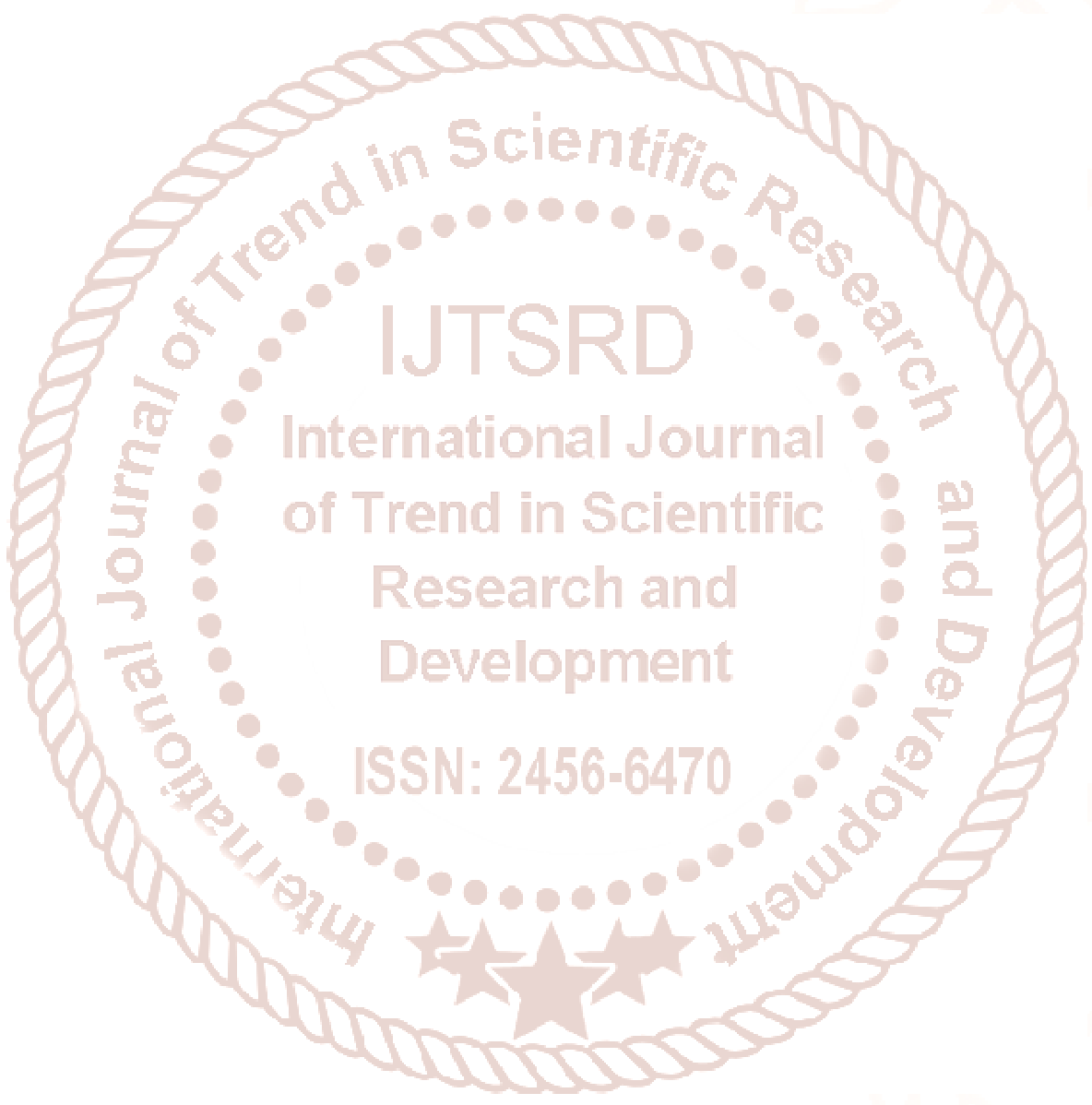

